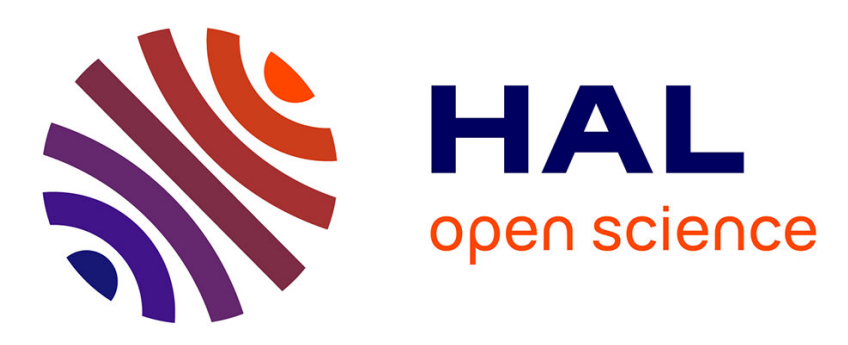

\title{
Tracking maritime cooperative target with real time motorized SAR antenna
}

Nicolas Castet, Olivier Ruault Du Plessis, Jean-Francois Nouvel, Sébastien Angelliaume

\section{- To cite this version:}

Nicolas Castet, Olivier Ruault Du Plessis, Jean-Francois Nouvel, Sébastien Angelliaume. Tracking maritime cooperative target with real time motorized SAR antenna. 2019 International Radar Conference (RADAR 2019), Sep 2019, TOULON, France. 10.1109/RADAR41533.2019.171284 . hal02470900

\section{HAL Id: hal-02470900 https://hal.science/hal-02470900}

Submitted on 2 Nov 2021

HAL is a multi-disciplinary open access archive for the deposit and dissemination of scientific research documents, whether they are published or not. The documents may come from teaching and research institutions in France or abroad, or from public or private research centers.
L'archive ouverte pluridisciplinaire HAL, est destinée au dépôt et à la diffusion de documents scientifiques de niveau recherche, publiés ou non, émanant des établissements d'enseignement et de recherche français ou étrangers, des laboratoires publics ou privés.

\section{(c)(1)}

Distributed under a Creative Commons Attribution| 4.0 International License 


\section{TRACKING MARITIME COOPERATIVE TARGET WITH REAL TIME MOTORIZED SAR ANTENNA}

\author{
Nicolas Castet \\ DEMR, ONERA \\ F-13661 Salon Cedex AIR - FRANCE \\ Nicolas.Castet@,onera.fr \\ Jean-François Nouvel \\ DEMR, ONERA \\ F-13661 Salon Cedex AIR - FRANCE \\ Jean-francois.Nouvel@onera.fr
}

\author{
Olivier Ruault du Plessis \\ DEMR, ONERA \\ F-13661 Salon Cedex AIR - FRANCE \\ Olivier.Ruault du Plessis@onera.fr \\ Sébastien Angelliaume \\ DEMR, ONERA \\ F-13661 Salon Cedex AIR - FRANCE \\ Sebastien.Angelliaume@onera.fr
}

\begin{abstract}
Since 2017, ONERA uses 2-axes motorized antenna to collect SAR images on various geometric acquisitions. Recently, a system has been developed to track maritime targets using the automatic identification system to follow them during illumination time. This paper describes each component of the system and presents all design and implementation steps.
\end{abstract}

Keywords - SETHI, SAR, Cooperative maritime target, Prediction, AIS

\section{INTRODUCTION}

SETHI platform [1][2] is an airborne system developed by ONERA dedicated to remote sensing (Fig.1). Recently, a special effort has been done to implement into the platform a new real time component to track maritime target. It is based on the use of the automatic identification system (AIS) emitted by cooperative vessel. Real time mobile target tracking is a technological and scientific challenge. Nevertheless, it is required for some scientific applications, like ultra-high spatial resolution (UHR) imagery or acquisition over the full $360^{\circ}$ of the azimuth range.

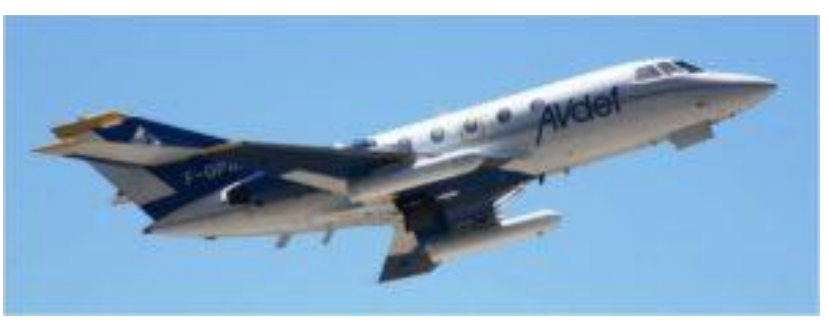

Fig. 1: SETHI platform

This paper is dedicated to the presentation of this new component into the SETHI airborne platform. The process sequencing is summarized in Fig.2, showing the four fundamental steps.

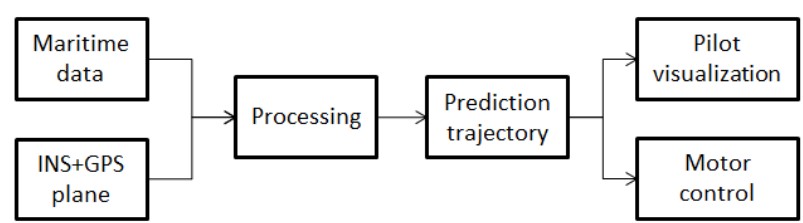

Fig. 2: Process sequencing to track cooperative target

This paper is organized as follow: Section II presents the X-band UHR SAR sensor, Section III details the AIS protocol (Fig.2 - step 1). Target selection (Fig.2 - step 2) and trajectory prediction (Fig.2 - step 3) are described in Section IV. The representation of flight assistance trajectories is proposed in section V (Fig.2 - step 4). Finally, validations are discussed in Section VI.

\section{X BAND SYSTEM AND ANTENNA MOTORIZATION}

SETHI is equipped with a high-performing X-band radar sensor (see Fig.3), with the main following characteristics:

- Carrier frequency $10 \mathrm{GHz}$.

- Dual polarization (H or V on transmit).

- Transmitted peak power $8 \mathrm{~kW}$.

- $\quad$ Large transmitted bandwidth, up to $4 \mathrm{GHz}$.

- 2-axes Tx and Rx motorized parabolic antennas.

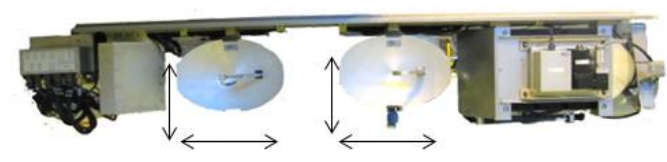

Fig. 3: Integration X-Band system in pod

Usually, SETHI collects SAR imagery on stripmap or spotlight mode. The last one can be associated with linear or circular trajectory (Fig.4).
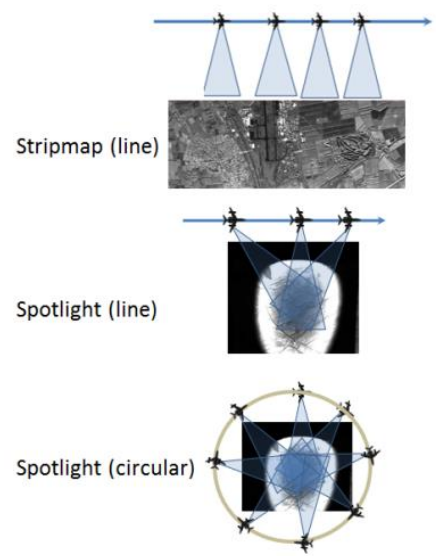

Fig. 4: Three radar modes geometries

For the purpose of maritime target observation we usually favored circular trajectories to allow SAR imagery over the full $360^{\circ}$ of the azimuth range. A dedicated 
antenna pointing algorithm has been designed to compensate in real time the aircraft's attitude during acquisition. The control frequency of the antennas is 100 $\mathrm{Hz}$ with a displacement specification of $45 \%$ on azimuth and $7 \%$ on elevation. The motion compensation is performed using information given every five milliseconds $(200 \mathrm{~Hz})$ by an onboard inertial unit. The accuracy of this information is $0.001^{\circ}$.

\section{SHIP RECOGNITION BY AIS}

The automatic identification system (AIS) [3] uses a transponder that transmits and receives protocol in VHFband. It includes the identification number of the vessel (MMSI), GPS information, ship velocity and attitude, among other.

\begin{tabular}{|c|c|c|c|c|}
\hline $\begin{array}{c}\text { Maritime Mobile Service } \\
\text { Identity (MMSI) }\end{array}$ & Longitude & Latitude & Heading & Speed \\
\hline
\end{tabular}

Table 1: Data used in an AIS frame

There are two types of AIS system, class A and B. For class $\mathrm{A}$, the range is about $20 \mathrm{NM}$. For class $\mathrm{B}$, the range is more limited between 5 and $10 \mathrm{NM}$. In addition, the message is transmitted with a frequency of approximately $30 \mathrm{~s}$ with class B and $10 \mathrm{~s}$ with class A. The low emission information rate is the first main constraint for the purpose of real time trajectory prediction. As example, for commercial vessels progressing at 15 knots (around $8 \mathrm{~m} / \mathrm{s}$ ), the resulting displacement between two transmitted AIS signal is about $150 \mathrm{~m}$ (assuming class B).

The new tracking system presented herein doesn't emit any AIS signal, it is only a receiver system composed by an omni-directional VHF antenna installed below the aircraft. Assuming a flight level of $15000 \mathrm{ft}$, the onboard system allows collecting cooperative signal emitted by vessel more than $30 \mathrm{NM}$ away. ONERA developed a modular and reliable system, called "ExaAIS", which is able to read AIS information from serial port and transferring data on Ethernet network. This software is based on open source library for decoding maritime automatic identification system messages "libAIS", python project.

\section{TARGET SELECTION AND PREDICTION}

Once a maritime target has been selected (identified by its MMSI), a software has been designed to predict the trajectory that will be followed by the vessel. It is called "ExaTRAJ" and relies on the use of the boat's previous position to interpolate its future localization. The following information is displayed onboard (Fig. 5):

- All marine cooperative targets on a map.

- Current aircraft position.

- Selection of a single target of interest.

- Plot of predicted trajectories.

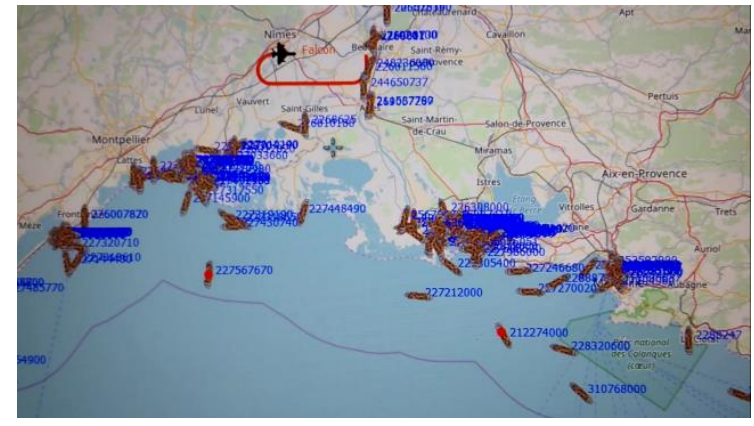

Fig. 5: Screenshot of the software interface

This software "ExaTRAJ" collects network data provided by "ExaAIS". Moreover, it process information provided by the aircraft inertial navigation system to display all this information on a single screen.

After selecting a target of interest, we have to predict the beginning of the ship trajectory, depending on the localization and the velocity of both the vessel and the aircraft. This prediction is based on the principle of dead reckoning [4] which consists of estimating one's current position using past information of localization, velocity and heading. One approach is to implement this equation.

$$
\mathrm{P}_{\mathrm{t}}=\mathrm{P}_{0}+\mathrm{V}_{0} \mathrm{~T}
$$

Where $\mathrm{P}_{0}$ is the received initial point, $\mathrm{V}_{0}$ the speed of the boat and $\mathrm{T}$ is the prediction time, usually one second. $P_{t}$ is the prediction point that can be determined. $P_{t}$ becomes $\mathrm{P}_{0}$ until $\mathrm{n}$ prediction time of duration $\mathrm{T}$ is reached. A small localization error spreads with each calculation of a new prediction.

The accuracy of the prediction is improved by using Vincenty's formulae [5][6] and more particularly, the first method of locating to a given distance and a direction from another point. This solution is based on the assumption that the figure of the Earth is an oblate ellipsoid and iterative method. Coupled with an AIS information refresh rate of about $30 \mathrm{~s}$, this procedure is accurate to within a few meters.

From the predicted trajectory of the target and on the same prediction principle, the circular trajectory of the aircraft is estimated as a function of the antenna pointing with a constant distance between the radar and the vessel. The main requirement that has to be fulfilled is that the target has to be kept at the center of the radar beam throughout the acquisition duration. This results in a solenoid pattern as displayed Fig. 6.

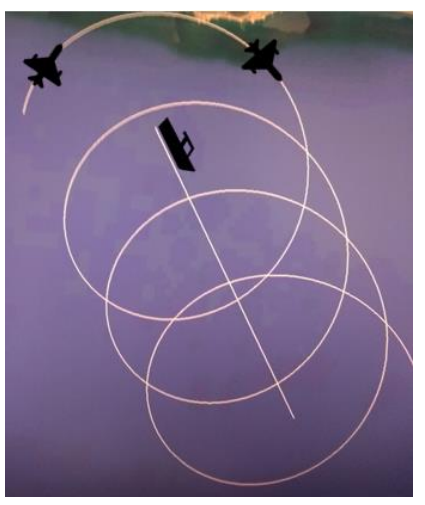

Fig. 6: Sliding circular path "Solenoid" 
During the acquisition, the software predicts the future position of the boat every second. This new position of the region of interest is transmitted to the antenna pointing device, in real time.

\section{Pilot Visualization}

Even though the aircraft trajectory shown in Fig. 6 is fairly simple to display on the flying screen, in practical case, it is not directly useful for the pilot. Indeed, the inflight conditions imply that the pilot has to compensate the aircraft attitude and needed display is more complex than a simple line. Thus, a corridor has been added to the required trajectory. It consists of parallel tracks informing the pilot about the maximum acceptable displacement. An illustration is given in Fig. 7, where one can observe the predicted aircraft trajectory (see the solenoid with corridor, in blue). The blue line at the top of the figure gives to the pilot the initial heading to enter into the solenoid. The actual aircraft's trajectory is plotted in red. The historical trajectory of the boat is plotted in yellow.

All developments were done with Qt and Marble / QML libraries with Windows and Linux compatibility.

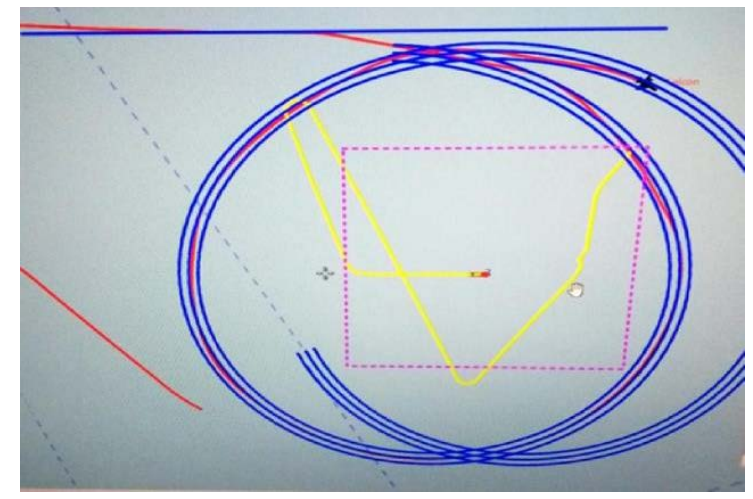

Fig. 7: Cooperative opportunity target

\section{RESULT IN OPERATIONAL CONTEXT}

The software development presented in this paper has been used during an experimental campaign of acquisition in the Mediterranean Sea. A target of interest has been imaged by the X-band sensor [7], the flying trajectory of the remote sensing system has been estimated in real time as illustrated in Fig. 8. In this figure, the aircraft is displayed in green and its actual trajectory is in red. The boat is near the center of the solenoid, the antenna pattern is also shown as well as the radar line of sight. The solenoid has an approximate duration of $10 \mathrm{mn}$. The target velocity is around $7 \mathrm{~m} / \mathrm{s}$, resulting in a total displacement during the acquisition of more than $4200 \mathrm{~m}$.
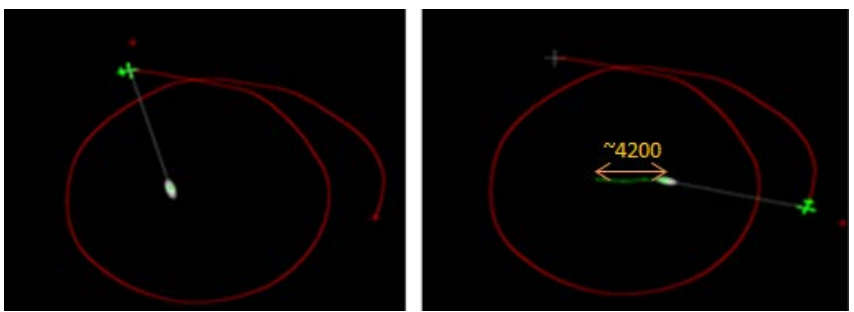

Fig. 8: Illustration of a full solenoid trajectory in an operational context.

\section{CONCLUSION}

Airborne SAR acquisition campaign dedicated to tracking cooperative maritime target has been performed in the Mediterranean Sea and validated the newly developped airborne guidance algorithms presented in this paper. The SETHI platform is now equipped with a new system allowing real time tracking of maritime target by the Xband SAR sensor. It relies on the use of AIS signal emitted by boat and received onboard. Software programs allow the processing of AIS and GPS data and perform a prediction of the vessel's trajectory. A spotlight mode predictive trajectory for the aircraft is then estimated and displayed for the pilot. Results in operational context have shown that the application method is consistent with ultrahigh spatial resolution imagery and allows collecting radar dataset over the full $360^{\circ}$ of the azimuth range.

In short term, some suggestions for improvements are being analyzed, like for example, a dynamic display of the solenoid trajectory by half-circle and a merging of both softwares - ExaAIS and ExaTraj.

\section{ACKNOWLEDGMENT}

Authors thank the DEMR/EXA and DEMR/TERE Research Units for their useful participation in data collection and processing.

\section{REFERENCES}

[1] Angelliaume, S.; Ceamanos, X.; Viallefont-Robinet, F.; Baqué, R.; Déliot, P.; Miegebielle, V. Hyperspectral and Radar Airborne Imagery over Controlled Release of Oil at Sea. Sensors 2017, 17, 1772.

[2] R. Baqué et al., "SETHI/RAMSES-NG new performances of the flexible multi-spectral airborne remote sensing research platform," International Conference on Radar Systems (Radar 2017), Belfast, 2017, pp. 1-4.

[3] https://en.wikipedia.org/wiki/Automatic identification system/

[4] https://en.wikipedia.org/wiki/Dead reckoning

[5] C. M. Thomas and W. E. Featherstone , "Validation of Vincenty's Formulas for the Geodesic Using a New Fourth-Order Extension of Kivioja's Formula", Journal of Surveying Engineering, Vol. 131, Issue 1 (February 2005), pp. 20-26.

[6] W. Kazimierski, "Problems of data fusion of tracking radar and AIS for the needs of integrated navigation systems at sea," 2013 14th International Radar Symposium (IRS), Dresden, 2013, pp. 270-275

[7] H. M. J. Cantalloube, L. Pastore-Guyonvarch, P. Martineau, J. Martinot-Lagarde, N. Castet and H. Oriot, "Maritime circular SAR processing issues: Antenna pattern verification, sea-clutter analysis \& ship image autofocus," EUSAR 2018; 12th European Conference on Synthetic Aperture Radar, Aachen, Germany, 2018, pp. 1-5. 